Cite this: Lab Chip, 2013, 13, 267

\title{
Programmable active droplet generation enabled by integrated pneumatic micropumps
}

\author{
Yong Zeng, *abc Mimi Shin ${ }^{\mathrm{ab}}$ and Tanyu Wang ${ }^{\mathrm{ab}}$
}

Received 8th August 2012, Accepted 19th October 2012

DOI: 10.1039/c2lc40906b

www.rsc.org/loc

\begin{abstract}
In this work we have investigated the integrated diaphragm micropump as an active fluidic control approach for the on-demand generation of droplets with precisely defined size, frequency and timing. In contrast to valve-actuated devices that only modulate the flow of the dispersed phase being continuously injected, this integrated micropump allows the combination of fluidic transport and modulation to achieve active control of droplet generation. A distinct characteristic of this method compared to the valve modulated droplet formation processes is that it enables independent control of droplet generation frequency by adjusting the pumping frequency and droplet size by flow conditions. We also demonstrated the generation of complex droplet patterns through programming the pumping configurations and the application to multi-volume digital PCR for precise and quantitative detection of genetic targets. Overall, our results suggest that the pump-based droplet microfluidics provide a robust platform for programmable active droplet generation which could facilitate the development of high-performance chemical and biological assays.
\end{abstract}

\section{Introduction}

Physical compartmentalization of chemical and biological assays has been long recognized for improving analysis/ processing performance, such as by enabling highly efficient reactions and sensitive detection, due to small volumes (femto-to nanolitre scales) and massive parallelism. ${ }^{1-4}$ Compared with early methods, the introduction of microfluidics revolutionizes the field by enabling the controllable generation of monodisperse droplets, automated fluidic manipulation and the high-throughput readout of information carried by discrete droplets. ${ }^{5,6}$ Thus, droplet microfluidics, an emerging platform alternative to continuous microsystems, has attracted increasing interest in developing novel tools for high-performance chemical and biological applications. ${ }^{7-12}$

Droplet microfluidics has been operated largely in passive fashion, such as droplet generation by flow focusing and crossflow shearing ${ }^{13}$ and downstream manipulation based on hydrodynamic interactions of droplets. ${ }^{14}$ Elucidation of complex and dynamic biological processes poses an urgent need of abilities to perform more complicated operations, including reagent dosing, mixing, splitting, extraction, detection and sorting, each of which would ideally require precise and automated control in space and time. Increasing efforts have been invested into understanding the underlying science and

${ }^{a}$ Department of Chemistry, University of Kansas, Lawrence, KS 66045, USA. E-mail: yongz@ku.edu; Fax: +1 (785) 864-5396; Tel: +1 (785) 864-8105

${ }^{b}$ Ralph $N$ Adams Institute of Bioanalytical Chemistry, University of Kansas, Lawrence, KS 66045, USA

${ }^{c}$ Bioengineering Program, University of Kansas, Lawrence, KS 66045, USA engineering principles of droplet microfluidics ${ }^{5,15-18}$ and exploring new approaches and devices to address the challenges in biological and medical applications ${ }^{10,19-21}$. For example, microfluidic circuits have been demonstrated for droplet injection, ${ }^{22}$ steering, ${ }^{23}$ trapping and release, ${ }^{24}$ synchronization, ${ }^{25}$ fusion, ${ }^{26}$ and sorting, ${ }^{27}$ leading to a much broader spectrum of applications, including digital $\mathrm{PCR},{ }^{28}$ protein engineering and direct evolution, ${ }^{29}$ and drug screening. ${ }^{30}$

As droplet microfluidics evolves, precise and controllable droplet generation has been recognized as an important component in developing droplet-based platforms for biological and medical applications. Droplet formation not only directly impacts on the performance of stochastic or ondemand droplet partition of targets, ${ }^{20,31}$ but also influences the downstream droplet manipulation, such as selective pairing and fusion. ${ }^{32-35}$ A variety of methods based on different mechanisms have been investigated to achieve controllable and eventually programmable droplet formation. For instance, piezoelectric actuators have been integrated with droplet generators to allow for precisely controlled droplet generation. ${ }^{34,36,37}$ Although this approach offers the drop ondemand capability, device fabrication and system operation are relatively complicated and require sophisticated equipment. Electrowetting provides a means for droplet formation and manipulation via controlling interfacial forces. ${ }^{35,38}$ However, this method suffers from some limitations, such as high susceptibility to the physical properties of samples and devices (e.g. capacitance and dielectric strength). ${ }^{38}$ Pneumatic valves fabricated by soft lithography have been demonstrated to be a promising technique for controlled droplet forma- 
tion. ${ }^{18,31-33}$ An advantage of this approach is its inherent amenability with large-scale microfluidic integration and automation. However, current valve-based methods rely on using external pumps to continuously flow the dispersed phase while actuating the integrated valves to modulate the passive droplet formation in microchannels.

In contrast to those valve-actuated devices, here we report on using an integrated valve-based diaphragm pump to control both fluidic transport and flow modulation of the dispersed phase for active droplet generation on demand. This method has been reported in our previous work for applications in single cell genetic analysis. ${ }^{20,21}$ However, this method has not been systematically studied to understand the droplet formation process driven by the on-chip pumping. Its applications to programming droplet generation have not been explored either. In this work we have systematically investigated the effects of pulsatile on-chip pumping and flow conditions on droplet formation in an eight-channel microfluidic T-junction droplet generator. A distinct characteristic of this method compared to valve modulated droplet formation processes is its ability to independently control droplet generation frequency by adjusting the pumping frequency and droplet size by flow conditions. Such an ability to precisely control the frequency and timing of droplet formation is advantageous in developing downstream droplet manipulation functionalities, such as selective droplet pairing and merging. ${ }^{32-35}$ Using the method, we also demonstrated the generation of complex droplet patterns through computer programming the pumping sequences and the application to multi-volume digital PCR for precise and quantitative detection of genetic targets.

\section{Experimental}

\section{Device fabrication and assembly}

The 8-channel microfluidic droplet generator was a four-layer, hybrid device composed of three glass layers and a thin poly(dimethylsiloxane) (PDMS) membrane (Fig. 1A). The valve seat structure was first lithographically fabricated on the top side of a BOROFLOAT ${ }^{\mathbb{R}}$ glass wafer. Using backside alignment lithography, the droplet generators were fabricated on the bottom of the same wafer in register with the pump structure. Fluidic and interlayer via holes were drilled and the wafer was thermally bonded to a clean substrate to enclose the microchannels. A microfabricated glass manifold for pneumatic control was aligned and reversibly bonded to the valve seat structure on top of the droplet generator chip through a $200 \mu \mathrm{m}$ PDMS membrane to form the on-chip micropump. The PDMS membrane and glass chips were exposed to UV ozone for $2 \mathrm{~min}$. right before the assembly to enhance the bonding strength.

\section{Droplet generation}

The hydrophilic glass channels were treated with a $0.1 \%$ solution of octadecyltrichlorosilane (OTS, Sigma-Aldrich) in dry toluene for $10 \mathrm{~min}$ to make the channel surfaces highly

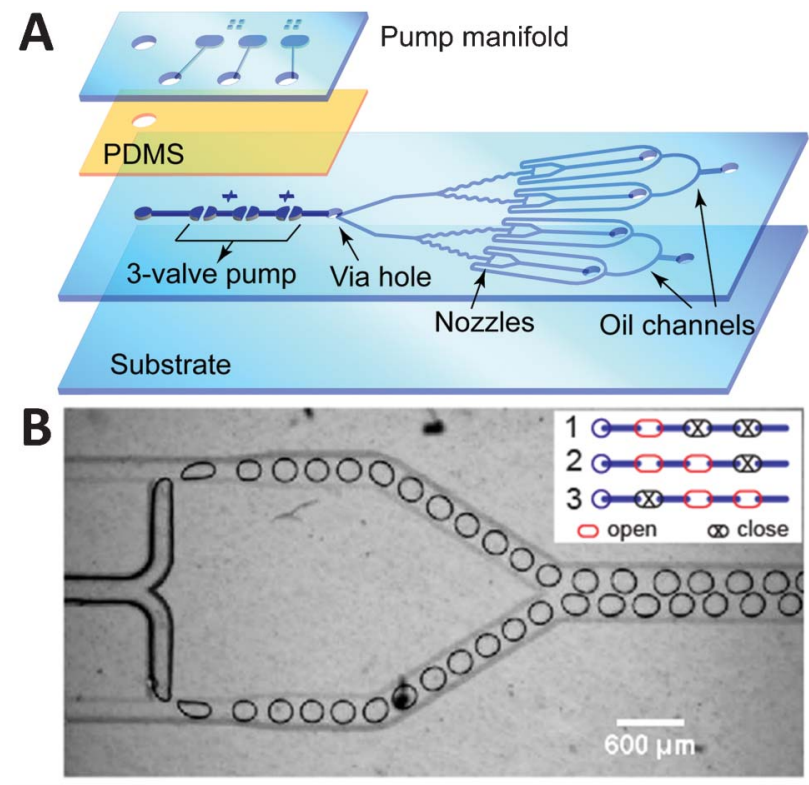

Fig. 1 (A) Exploded view of a glass/PDMS/glass hybrid 8-channel microfluidic droplet generator array device integrated with a pneumatically controlled three-valve micropump. (B) Microscopic photo of parallel generation of uniform water-in-oil droplets of $\sim 2 \mathrm{nl}$ in two of eight T-junction generators in a device. A three-step actuation sequence (inset) was carried out with the actuation periods of 45,40 , and $40 \mathrm{~ms}$, respectively. A pressure of $40 \mathrm{kPa}$ was applied for pneumatic actuation.

hydrophobic. The carrier oil contains 39.8\% (w/w) DC 5225C Formulation Aid (Dow Chemical Co., Midland, MI), 30\% (w/w) DC 749 Fluid (Dow Chemical Co.), 30\% (w/w) AR20 silicone oil (Sigma-Aldrich), and $0.2 \%(\mathrm{w} / \mathrm{w})$ Triton X-100 surfactant (Sigma-Aldrich). A custom PLEXIGLAS ${ }^{\circledR}$ manifold was assembled with the MEGA device to allow oil infusion and droplet collection. A syringe pump (Harvard Apparatus, Holliston, MA) was used to continuously inject carrier oil into the MEGA device at various flow rates. The on-chip 3-valve diaphragm pump was pneumatically actuated by a homemade solenoid valve system to pump the aqueous solution through the channels. A Welch dry vacuum pump was used to supply constant vacuum $(-80 \mathrm{kPa})$ for valve actuation. The pressure was varied as specified in the text. The pumping was conducted in a three-step fashion (Fig. 1B, inset) under the control of a program written in LabVIEW (National Instruments, Austin, TX). Pumping parameters were specified in the main text. Droplets were collected in $0.5 \mathrm{ml}$ PCR tubes.

\section{Droplet PCR and fluorescence imaging}

All oligonucleotides used in this study were ordered from IDT. PCR reagents were obtained from Life Technologies (Carlsbad, CA). PCR mix contained $1 \times$ Platinum $^{\circledR}$ Taq DNA polymerase buffer with $3 \mathrm{mM} \mathrm{MgCl}$, $0.2 \mathrm{mM}$ dNTPs, $0.4 \mu \mathrm{g} \mu \mathrm{l}^{-1}$ heatinactivated BSA (Sigma), $0.4 \mu \mathrm{M}$ each of primers (reverse: 5'-TAAGCACGAACTCAGCCAGAACGA-3'; forward: 5'-CGCGATATGCTGCGCTTGCT-3'), $0.15 \mathrm{U}^{\prime-1} \mathrm{l}^{-1}$ Platinum $^{\circledR}$ Taq DNA polymerase, $1 \times$ EvaGreen $^{\mathrm{TM}}$ dye (Biotium, Hayward, CA), and $\lambda$-DNA (New England Biolabs). $\lambda$-DNA samples were prepared from the $10 \mu \mathrm{g} \mathrm{ml}^{-1}$ stock by diluting the concentration to the 
order of $10 \mathrm{pg} \mathrm{ml}^{-1}$. Thermal cycling was carried out with a 2 min hot start at $95{ }^{\circ} \mathrm{C}$, and 40 cycles of $95{ }^{\circ} \mathrm{C}$ for $30 \mathrm{~s}, 60{ }^{\circ} \mathrm{C}$ for $30 \mathrm{~s}, 72{ }^{\circ} \mathrm{C}$ for $30 \mathrm{~s}$ in a MasterCycler ${ }^{\mathbb{B}}$ nexus thermocycler (Eppendorf). For fluorescence imaging analysis, droplets were pipetted onto a glass slide treated with OTS (Sigma) and slowly spread out to form a monolayer of droplets. Both bright field and fluorescence images were taken using a Zeiss Axiovert A1 inverted fluorescence microscope equipped with a LED excitation light source (Thorlabs, Newton, NJ). The digital images were processed and analyzed using Image (NIH, http://rsbweb.nih.gov/ij/) to measure the size and fluorescence intensity of individual droplets. ${ }^{39}$ Touching droplets and droplet split-up or coalescence caused by pipetting can interfere with the droplet size measurement. A confidence interval of $99 \%$ was adopted to eliminate the outliers.

\section{Results and discussion}

An important consideration in designing new micropumpbased generators for high-speed droplet production is to increase the channel multiplicity while minimizing the dimensions and number of micropumps. We found that the micropump which was reported for the four-channel device ${ }^{20}$ is able to operate eight parallel T-shaped droplet generators without changing the pump size (Fig. 1A). Fig. 1B shows a typical microscopic photo of the parallel generation of uniform water-in-oil droplets of $\sim 2 \mathrm{nl}$ using the 8-channel device. A three-step actuation sequence (45/40/40 ms) was conducted (Fig. 1B inset) to increase the pumping frequency, and hence the droplet formation rate, compared to the fourstep actuation method used before. This 8-channel design should allow construction of generator arrays with at least doubled channel density and droplet generation capacity with respect to the 4 -channel design. ${ }^{20}$ Unless specified, the 8-channel MEGA device developed here was used to investigate the active droplet formation driven by on-chip pumping.

We first studied the effects of the flow rate of the continuous oil phase as it plays a critical role in passive microfluidic droplet generation. ${ }^{13}$ Fig. 2A plots the droplet formation frequency $\left(f_{\text {droplet }}\right)$ and droplet size $\left(R_{\text {droplet }}\right)$ as a function of the oil flow rate in each T-junction $\left(Q_{\text {oil }}\right)$ under an actuation sequence of $40 /$ $50 / 40 \mathrm{~ms}$ and a pressure of $35 \mathrm{kPa}$. Four distinct regimes were identified as $Q_{\text {oil }}$ increases from 0.2 to $5 \mu \mathrm{min}^{-1}$. At the low oil flow rate in the regime (a), large uniform droplets were periodically formed, as shown in Fig. 2B (a). Interestingly, $f_{\text {droplet }}$ was much lower than the pumping frequency $\left(f_{\text {pump }}\right)$ of $7.69 \mathrm{~Hz}$. $f_{\text {droplet }}$ increases along with $Q_{\text {oil }}$ while the droplet size $R_{\text {droplet }}$ decreases. Such behavior was found to be characteristic of confined droplet break-up in T-junctions. ${ }^{15,16}$ Microscopic visualization also observed a droplet break-up process which resembles that of passive cross-flow droplet formation. ${ }^{4}$ These results indicate that the droplet break-up within the regime (a) is predominantly governed by the interaction of viscous shear stresses and interfacial tension between the two immiscible phases and that the force generated by mechanical pump actuation plays a minor role. ${ }^{18}$
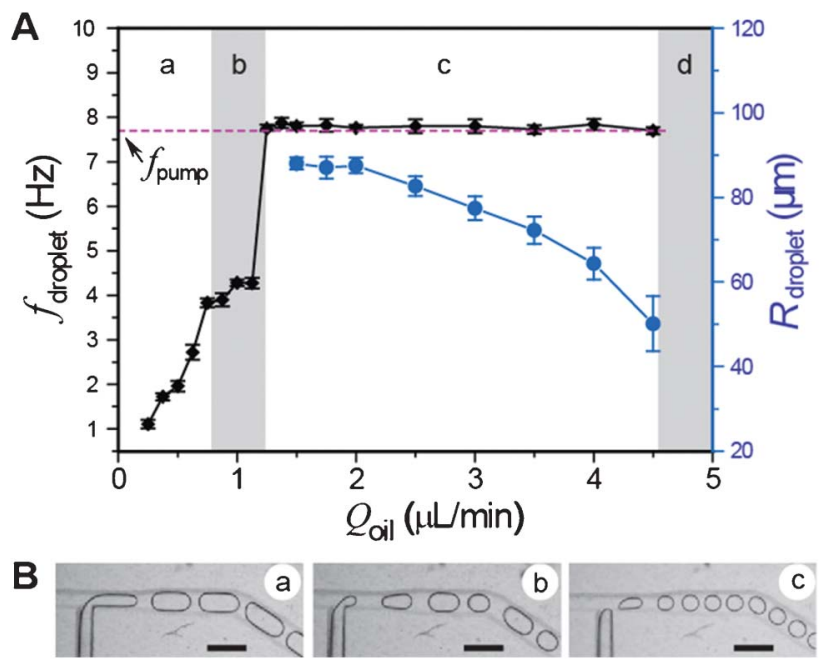

Fig. 2 (A) Plot of the droplet formation frequency $\left(f_{\text {droplet }}\right)$ and droplet radius $\left(R_{\text {droplet }}\right)$ as a function of the oil flow rate in each T-junction $\left(Q_{\text {oil }}\right)$ under an actuation sequence of 40/50/40 ms and a pressure of $35 \mathrm{kPa}$. The dashed line indicates the pumping frequency $\left(f_{\text {pump }}\right)$ of $7.69 \mathrm{~Hz}$. (B) Images of different droplet formation behaviors observed in the regimes identified in (A). The scale bars are $600 \mu \mathrm{m}$

As $Q_{\text {oil }}$ was further increased, a transition regime (b) occurred in which droplet generation lacks both periodicity and size uniformity, as seen in Fig. 2B (b). This transition window in $Q_{\text {oil }}$ is narrow, usually less than $0.5 \mu \mathrm{min}^{-1}$. A sharp change of droplet generation behavior was observed when $f_{\text {droplet }}$ was tuned to a threshold value of $1.3 \mu \mathrm{lmin}^{-1}$ (Fig. 2A (c)). Within this regime of a wide range of $Q_{\text {oil }}$, the use of active on-chip pumping enables stable generation of uniform droplets, as exemplified in Fig. 2B (c). Several unique phenomena were observed in this regime. First, the droplet generation synchronizes with the pumping frequency, which can be attributed to the pulsatile nature of on-chip pumping. We found that the aqueous solution is pulled back at the end of each pumping stroke, which in fact increases the shearing force to facilitate the break-up of the droplets. As a result, the pump actuation modulates the process of droplet formation. This phenomenon has been found to be characteristic of droplet generation driven by pulsatile on-chip pumping; it is not only observed for the cross-flowing mode in the T-junction device used here, but also for the flow-focusing mode in a 4-channel droplet generator ${ }^{40}$ and a 96-channel device with a different pump structure. ${ }^{20}$ Second, $f_{\text {droplet }}$ is independent of $Q_{\text {oil }}$, which indicates the dominance of the forces caused by pumping over the viscous shear stresses that depend on the flow parameters. Third, the droplet size can be independently tuned by varying $Q_{\text {oil }}$. Increasing $Q_{\text {oil }}$ leads to higher back pressure which suppresses the pneumatic actuation of the valves and thus reduces the flow rate of the aqueous phase being pumped into the channels. ${ }^{41}$ Eventually the droplet generators will stop working when the back pressure is larger than the pressure that the pump is able to generate, as shown in Fig. 2A (d). Although the droplet size variation was larger at 
higher $Q_{\text {oil }}$, the droplet formation was observed to remain synchronized with the pumping. These observations clearly demonstrate that on-chip pumping confers the ability to independently control frequency by adjusting pumping rate and droplet size by varying $Q_{\text {oil }}$. This unique property is in contrast to the passive droplet generation methods in which droplet generation frequency and droplet size are linked and sensitive to the flow conditions. ${ }^{13}$

Fig. 2 shows that a range of $Q_{\text {oil }}$ needs to be experimentally defined to ensure precisely controlled droplet generation with excellent size uniformity when using the on-chip pumping method. As expected, the $Q_{\text {oil }}$ range and the droplet sizes were found to be also affected by the pumping conditions, such as $f_{\text {pump }}$ and the pressure for pneumatic actuation. Fig. 3A shows that the threshold $Q_{\text {oil }}$ at which the droplet generation and the pumping start to synchronize is a roughly linear function of $f_{\text {pump }}$, especially at the high frequencies. At a fixed pressure (same as in Fig. 2A) within the range that we commonly used, increasing the pumping rate requires a higher oil flow rate to establish stable operation. Since the droplet size is also affected by $Q_{\text {oil }}$ (Fig. 2A), one should work at the oil flow rates away from the boundary conditions such that the adjustment of droplet generation frequency by varying $f_{\text {pump }}$ will require no changes of oil flow and thus have no effects on the droplet sizes. In addition to the oil flow rate, droplet sizes can be fine tuned by adjusting the actuation pressure. The droplet volume exhibits a peaking response to increasing actuation pressure under the conditions of pumping and oil flow that we commonly used for fast droplet generation. An example is shown in Fig. 3B with a pumping sequence of 45/45/45 ms and $Q_{\text {oil }}$ at $3 \mu \mathrm{min}^{-1}$. The experimental observations in Fig. 2 and 3 would provide informative guidance for exploring the proper conditions for independent and precise control of droplet frequency and size.

It is important to note that our results show distinct droplet generation behaviors from those of the mechanical actuationbased methods that use PDMS or piezoelectric valves. ${ }^{18,37}$ In a PDMS valve-based method, the dispersed phase is constantly driven by external pumps and a valve placed close to the droplet generation junction is periodically actuated to alter the
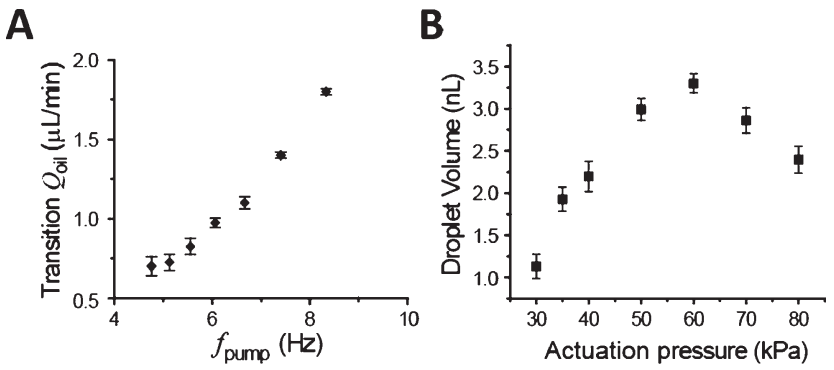

Fig. 3 Effects of the pumping conditions on active droplet generation. (A) Plot of the threshold $Q_{\text {oil }}$ as a function of the pumping frequency $\left(f_{\text {pump }}\right)$ obtained under an actuation pressure of $35 \mathrm{kPa}$. The duration for each actuation step was kept the same. (B) Plot of the droplet volume as a function of the actuation

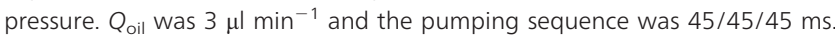

instantaneous flow rate of the dispersed phase. ${ }^{18}$ It was found that the forced droplet generation synchronizes with the natural droplet generation only if the actuation frequency is a multiple of the natural frequency. Otherwise irregular droplet formation will occur. In our method, synchronized droplet formation can be obtained at any pumping frequency as long as the oil flow rate falls in the regime (c) (Fig. 2 and 3A). Therefore, our studies suggest a different physical mechanism which should arise from the combination of pulsatile transport and modulation of the dispersed phase, in contrast to the valve-based devices that only modulate the flow being continuously injected. To fully elucidate the dynamics of droplet formation driven by the pulsing pumping, more experimental and theoretical studies are needed to capture the pictures of the impact of pump actuation on the droplet generation process including droplet growth, necking, and break-off. $^{5,42}$

Because the on-chip diaphragm pump directly delivers the dispersed phase in our device, pumping frequency or period is expected to be an important factor in determining the flow rate of the dispersed phase and thus the droplet size. As seen in Fig. 4A, the droplet size increases and then levels off as the pumping period is extended. Such behavior is consistent with the reported relationship between the flow rate and the actuation time for a microfabricated 3-valve pump. ${ }^{41}$ Adjusting the pumping period provides a means to control droplet generation that is more compatible with computer programming. The top photo in Fig. 4B shows a stream of two differently sized droplets that was formed by continuously alternating two pumping cycles of $150 \mathrm{~ms}$ and $105 \mathrm{~ms}$. In addition, the droplets can be formed on demand to yield more complex patterns, as demonstrated in the bottom photo of Fig. 4B. In this case, each repeating sequence consists of 7 steps: two consecutive pumping cycles of 130 and $165 \mathrm{~ms}, 100$ ms interval, $110 \mathrm{~ms}$ pumping, $300 \mathrm{~ms}$ interval, $150 \mathrm{~ms}$ pumping, and $500 \mathrm{~ms}$ interval. Clearly, the on-chip pumping method allows precise control of droplet generation frequency, size, and timing, which demonstrates its on-demand capability and programmability. Compared to the valve-based devices, our design eliminates the need for external driving pumps such as bulky syringe pumps for sample injection. This is particularly advantageous when handling multiple samples is needed because the on-chip pump is inherently amenable with microfluidic integration, automation and scale-up. ${ }^{6,20,43}$

In Fig. 5, we demonstrate that the ability to program droplet formation enables multi-volume droplet PCR (MVdPCR) for precise digital quantification of genetic targets. At a given target concentration, the droplet volume determines the average droplet occupancy, which is referred as the average concentration per droplet (copies/droplet). Therefore, MVdPCR provides a means to simultaneously carry out multiple measurements of a sample at various average concentrations per droplet, which is equivalent to the serial dilution measurements. Compared to serial dilution measurements by single-volume dPCR, MV-dPCR can improve the detection reproducibility by avoiding the random and/or system errors 


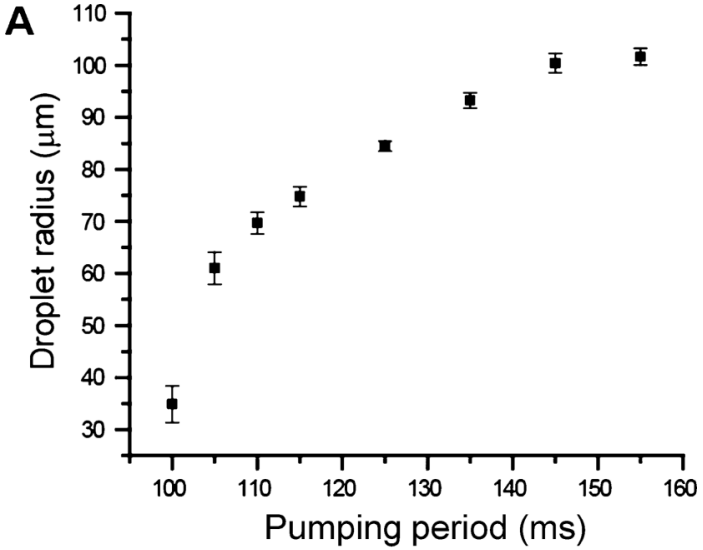

B
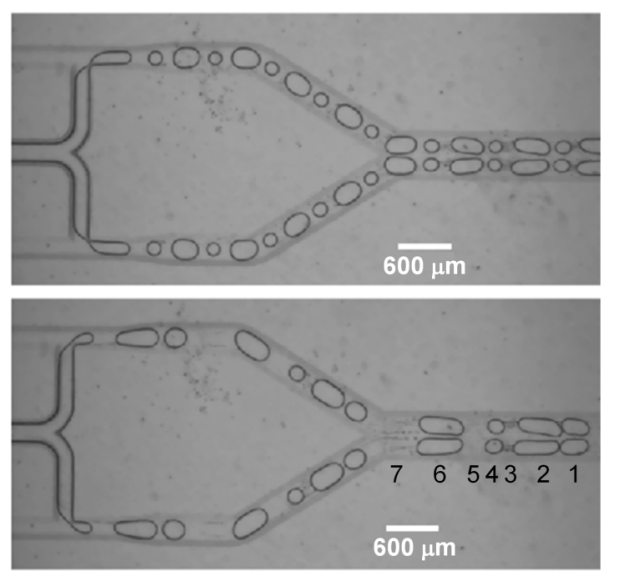

Fig. 4 Programmable droplet generation by controlling the pumping sequences. (A) Plot of droplet radius as a function of the pumping period. (B) The top photo shows a stream of two differently sized droplets formed by continuously alternating two pumping cycles of $150 \mathrm{~ms}$ and $105 \mathrm{~ms}$. The bottom photo shows on-demand generation of more complex patterns. In this case, each repeating sequence consists of 7 steps: $(1,2)$ two consecutive pumping cycles of 130 and $165 \mathrm{~ms}$, (3) $100 \mathrm{~ms}$ interval, (4) $110 \mathrm{~ms}$ pumping, (5) 300 ms interval, (6) 150 ms pumping, and (7) 500 ms interval. The actuation

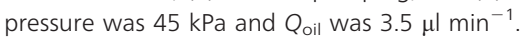

that arise from sample handling and measuring multiple samples in variable conditions. Moreover, it was found that MV-dPCR offers a wider dynamic range and better sensitivity than single-volume dPCR assays. ${ }^{44,45}$

Fig. 5A shows a representative fluorescence image for the end-point dPCR detection of a $\lambda$-DNA sample using a mixture of four differently sized droplets. In this case, droplets of 73$265 \mu \mathrm{m}$ in diameter were formed by using a pumping sequence of consecutive 7 cycles: one $165 \mathrm{~ms}$, one $140 \mathrm{~ms}$, two $120 \mathrm{~ms}$, and three $100 \mathrm{~ms}$ cycles. More droplets of smaller sizes were formed to increase the statistical power of the single molecule detection in the low copy number regime. ${ }^{45}$ As seen in the image, most of the large droplets turn fluorescent due to the high DNA concentration per droplet and an increasing number of droplets remain dark as the droplet sizes decrease. The dot plot in Fig. 5B shows the distribution of positive/ negative droplets versus the droplet size, providing an overview of the observations in Fig. 5A. To the right of the dot plot are
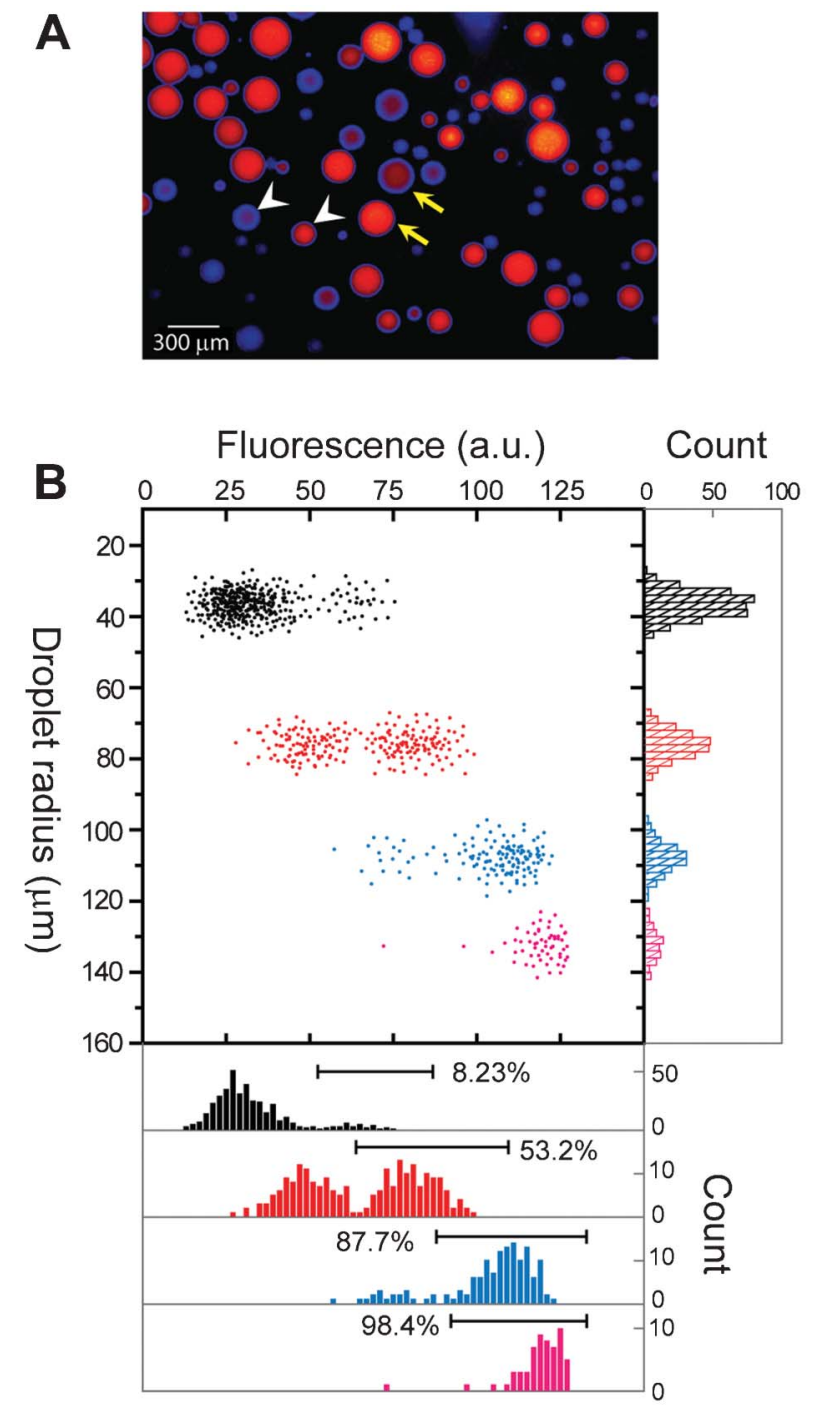

Fig. 5 Multi-volume droplet digital PCR (MV-dPCR). (A) A typical fluorescence image of the droplets after PCR thermal cycling. A mixture of four differently sized droplets of 73-265 $\mu \mathrm{m}$ in diameter was produced to statistically encapsulate $\lambda$-DNA molecules using a pumping sequence of consecutive 7 cycles: one $165 \mathrm{~ms}$, one $140 \mathrm{~ms}$, two $120 \mathrm{~ms}$, and three $100 \mathrm{~ms}$ cycles. The yellow arrows and white arrow heads highlight two pairs of droplets from two size categories to compare the positive and negative droplets. Other conditions were identical to those of Fig. 4. (B) A dot plot showing the distribution of positive and negative droplets versus the droplet size. To the right of the dot plot are the histograms of the size distribution for the four droplet groups. Below the dot plot are the histograms of fluorescence intensity which were gated to determine the percentage of positive events for each droplet group.

the histograms of size distribution for the four droplet groups. The relative standard deviation in diameter is determined to be lower than $5 \%$ except for the smallest droplet $(73.1 \mu \mathrm{m}$, $9.7 \%$ size deviation). The relatively large size deviation could be attributed to the large droplet nozzles $(200 \mu \mathrm{m}$ wide and 80 $\mu \mathrm{m}$ deep) which were found to be more suitable for uniform formation of large droplets. Optimizing the channel dimensions would further improve the size uniformity for small droplets. ${ }^{13,46}$ The fluorescence intensity histograms of droplets 
Table $1 \mathrm{MV}$-dPCR measurements of $\lambda$-DNA concentration

\begin{tabular}{|c|c|c|c|c|c|}
\hline $\begin{array}{l}\text { Averaged droplet } \\
\text { diameter }(\mu \mathrm{m})\end{array}$ & $\begin{array}{l}\text { Number of } \\
\text { analyzed droplets }\end{array}$ & $\begin{array}{l}\text { Expected positive } \\
\text { droplets }(\%)\end{array}$ & $\begin{array}{l}\text { Observed positive } \\
\text { droplets }(\%)\end{array}$ & $\begin{array}{l}\text { Concentration } \\
\text { (copies/drop) }\end{array}$ & $\begin{array}{l}\text { Concentration } \\
\left(\text { copies } \mu l^{-1}\right)\end{array}$ \\
\hline 73.1 & 397 & 9.07 & 8.23 & 0.0858 & 419 \\
\hline 156.0 & 247 & 57.5 & 53.2 & 0.759 & 412 \\
\hline 215.7 & 140 & 91.3 & 87.7 & 2.09 & 398 \\
\hline 264.8 & 61 & 98.9 & 98.0 & 3.93 & 404 \\
\hline
\end{tabular}

(Fig. 5B, below the dot plot) were gated to determine the percentage of positive events, which allows us to calculate the average DNA concentration per droplet and the bulk concentration (copies $\mu \mathrm{l}^{-1}$ ) for each droplet size based on the Poisson distribution. ${ }^{39,20}$ As summarized in Table 1, the observed positive droplet percentages agree with the expected values and the average concentrations determined from different droplet sizes agree with each other quantitatively. The "most probable" concentration is calculated to be $456 \pm 28$ copies $\mu^{-1}$ by combining the results from the four droplet sizes, which is consistent with the expected copy number concentration of 460 copies $\mu^{-1}$ (i.e., $25 \mathrm{pg} \mathrm{ml}^{-1}$ ). This calculation based on the most probable number (MPN) theory provides more precise quantification of targets than that measured from single droplet size. ${ }^{45,47}$ These results suggest that the MV-dPCR assay based on the pump-drive active droplet formation allows for precise digital quantification of genetic targets in a single measurement.

MV-dPCR has been demonstrated using SlipChips that contain microfabricated wells of different volumes. ${ }^{44,45}$ The SlipChip design has fixed compartment dimensions and can only accommodate a certain number of wells, which limits the dynamic range and sensitivity of dPCR assays. Our on-demand droplet method offers great flexibility in choosing the volume of reaction compartments and the range of compartment volumes to adjust sensitivity and dynamic range for different applications. Thus the pump-based microfluidic droplet generator should provide a versatile platform for accurate and sensitive MV-dPCR quantification of genetic targets of biological and clinical significance.

\section{Conclusions}

Here we have investigated pulsatile pumping for on-demand droplet generation using an 8-channel microfluidic droplet generator device integrated with a three-valve diaphragm pump. We have identified four regimes in droplet formation frequency as the flow rate of the continuous oil phase changes. In the regime c, where stable droplet generation is established, the droplet formation frequency is synchronized with the pumping rate over a wide range of oil flow rates while the droplet size can be independently adjusted by tuning the oil flow rate and pneumatic pressure. Such unique behavior suggests a distinct mechanism underlying the pump-driven droplet formation compared to that of the valve-actuated methods. We further demonstrated that the active pumping method allows for programming pumping sequences to achieve on-demand formation of complex droplet patterns. Such capability enables multi-volume droplet digital PCR for precise and quantitative detection of genetic targets. The studies reported here using the single-pump design would provide valuable guidance for devising highly integrated multipump microsystems capable of performing complex microfluidic functionalities, such as droplet synchronization, merging and mixing. . $^{72}$ Overall, our results suggest that pumpbased droplet microfluidics provide a robust platform for programmable active droplet generation, which could facilitate the development of high-performance chemical and biological assays.

\section{Acknowledgements}

The work was supported by the new faculty start-up funds from the University of Kansas. M.S. acknowledges a Bailey Scholarship from the Department of Chemistry at the University of Kansas.

\section{References}

1 B. Rotman, Proc. Natl. Acad. Sci. U. S. A., 1961, 47, 1981-1991.

2 D. Dressman, H. Yan, G. Traverso, K. W. Kinzler and B. Vogelstein, Proc. Natl. Acad. Sci. U. S. A., 2003, 100, 8817-8822.

3 B. T. Kelly, J. C. Baret, V. Taly and A. D. Griffiths, Chem. Commun., 2007, 1773-1788.

4 Y. Rondelez, G. Tresset, K. V. Tabata, H. Arata, H. Fujita, S. Takeuchi and H. Noji, Nat. Biotechnol., 2005, 23, 361-365.

5 T. Glawdel, C. Elbuken and C. L. Ren, Phys. Rev. E: Stat., Nonlinear, Soft Matter Phys., 2012, 85, 016323.

6 J. Kim, E. C. Jensen, M. Megens, B. Boser and R. A. Mathies, Lab Chip, 2011, 11, 3106-3112.

7 S. Y. Teh, R. Lin, L. H. Hung and A. P. Lee, Lab Chip, 2008, 8, 198-220.

8 X. Niu and A. J. Demello, Biochem. Soc. Trans., 2012, 40, 615-623.

9 R. Seemann, M. Brinkmann, T. Pfohl and S. Herminghaus, Rep. Prog. Phys., 2012, 75, 016601.

10 E. Brouzes, Methods Mol. Biol., 2012, 853, 105-139.

11 L. Malic, D. Brassard, T. Veres and M. Tabrizian, Lab Chip, 2010, 10, 418-431. 
12 S. Cho, D. K. Kang, J. Choo, A. J. deMello and S. I. Chang, BMB Rep., 2011, 44, 705-712.

13 G. F. Christopher and S. L. Anna, J. Phys. D: Appl. Phys., 2007, 40, R319-R336.

14 M. Prakash and N. Gershenfeld, Science, 2007, 315, 832-835.

15 G. F. Christopher, N. N. Noharuddin, J. A. Taylor and S. L. Anna, Phys. Rev. E: Stat., Nonlinear, Soft Matter Phys., 2008, 78, 036317.

16 P. Garstecki, M. J. Fuerstman, H. A. Stone and G. M. Whitesides, Lab Chip, 2006, 6, 437-446.

17 T. Glawdel, C. Elbuken and C. Ren, Lab Chip, 2011, 11, 3774-3784.

18 H. Willaime, V. Barbier, L. Kloul, S. Maine and P. Tabeling, Phys. Rev. Lett., 2006, 96, 054501.

19 S. Q. Gu, Y. X. Zhang, Y. Zhu, W. B. Du, B. Yao and Q. Fang, Anal. Chem., 2011, 83, 7570-7576.

20 Y. Zeng, R. Novak, J. Shuga, M. T. Smith and R. A. Mathies, Anal. Chem., 2010, 82, 3183-3190.

21 R. Novak, Y. Zeng, J. Shuga, G. Venugopalan, D. A. Fletcher, M. T. Smith and R. A. Mathies, Angew. Chem., Int. Ed., 2011, 50, 390-395.

22 A. R. Abate, T. Hung, P. Mary, J. J. Agresti and D. A. Weitz, Proc. Natl. Acad. Sci. U. S. A., 2010, 107, 19163-19166.

23 P. Abbyad, R. Dangla, A. Alexandrou and C. N. Baroud, Lab Chip, 2011, 11, 813-821.

24 M. G. Simon, R. Lin, J. S. Fisher and A. P. Lee, Biomicrofluidics, 2012, 6.

25 B. Ahn, K. Lee, H. Lee, R. Panchapakesan and K. W. Oh, Lab Chip, 2011, 11, 3956-3962.

26 L. Mazutis and A. D. Griffiths, Lab Chip, 2012, 12, 1800-1806.

27 T. Franke, S. Braunmuller, L. Schmid, A. Wixforth and D. A. Weitz, Lab Chip, 2010, 10, 789-794.

28 D. Pekin, Y. Skhiri, J. C. Baret, D. Le Corre, L. Mazutis, C. B. Salem, F. Millot, A. El Harrak, J. B. Hutchison, J. W. Larson, D. R. Link, P. Laurent-Puig, A. D. Griffiths and V. Taly, Lab Chip, 2011, 11, 2156-2166.

29 A. Fallah-Araghi, J. C. Baret, M. Ryckelynck and A. D. Griffiths, Lab Chip, 2012, 12, 882-891.
30 O. J. Miller, A. El Harrak, T. Mangeat, J. C. Baret, L. Frenz, B. El Debs, E. Mayot, M. L. Samuels, E. K. Rooney, P. Dieu, M. Galvan, D. R. Link and A. D. Griffiths, Proc. Natl. Acad. Sci. U. S. A., 2012, 109, 378-383.

31 R. Lin, J. S. Fisher, M. G. Simon and A. P. Lee, Biomicrofluidics, 2012, 6, 24103.

32 S. Zeng, B. Li, X. Su, J. Qin and B. Lin, Lab Chip, 2009, 9, 1340-1343.

33 F. Guo, K. Liu, X. H. Ji, H. J. Ding, M. Zhang, Q. A. Zeng, W. Liu, S. S. Guo and X. Z. Zhao, Appl. Phys. Lett., 2010, 97, 233701.

34 J. Shemesh, A. Nir, A. Bransky and S. Levenberg, Lab Chip, 2011, 11, 3225-3230.

35 H. Gu, C. U. Murade, M. H. G. Duits and F. Mugele, Biomicrofluidics, 2011, 5, 011101.

$36 \mathrm{~J} . \mathrm{Xu}$ and D. Attinger, J. Micromech. Microeng., 2008, 18, 065020.

37 A. Bransky, N. Korin, M. Khoury and S. Levenberg, Lab Chip, 2009, 9, 516-520.

38 R. B. Fair, Microfluid. Nanofluid., 2007, 3, 245-281.

39 L. B. Pinheiro, V. A. Coleman, C. M. Hindson, J. Herrmann, B. J. Hindson, S. Bhat and K. R. Emslie, Anal. Chem., 2012, 84, 1003-1011.

40 E. C. Jensen, Y. Zeng, J. Kim and R. A. Mathies, JALA Charlottesv Va, 2010, 15, 455-463.

41 W. H. Grover, A. M. Skelley, C. N. Liu, E. T. Lagally and R. A. Mathies, Sens. Actuators, B, 2003, 89, 315-323.

42 T. Glawdel, C. Elbuken and C. L. Ren, Phys. Rev. E: Stat., Nonlinear, Soft Matter Phys., 2012, 85, 016323.

43 E. C. Jensen, B. P. Bhat and R. A. Mathies, Lab Chip, 2010, 10, 685-691.

44 J. E. Kreutz, T. Munson, T. Huynh, F. Shen, W. Du and R. F. Ismagilov, Anal. Chem., 2011, 83, 8158-8168.

45 F. Shen, B. Sun, J. E. Kreutz, E. K. Davydova, W. Du, P. L. Reddy, L. J. Joseph and R. F. Ismagilov, J. Am. Chem. Soc., 2011, 133, 17705-17712.

46 N. R. Beer, B. J. Hindson, E. K. Wheeler, S. B. Hall, K. A. Rose, I. M. Kennedy and B. W. Colston, Anal. Chem., 2007, 79, 8471-8475.

47 J. E. Kreutz, T. Munson, T. Huynh, F. Shen, W. B. Du and R. F. Ismagilov, Anal. Chem., 2011, 83, 8158-8168. 